\title{
An effective technique for the conformable space-time fractional EW and modified EW equations
}

https://doi.org/10.1515/nleng-2017-0025

Received February 12, 2017; revised March 17, 2018; accepted April 7, 2018.

\begin{abstract}
The current work deals with the fractional forms of EW and modified EW equations in the conformable sense and their exact solutions. In this respect, by utilizing a traveling wave transformation, the governing space-time fractional models are converted to the nonlinear ordinary differential equations (NLODEs); and then, the resulting NLODEs are solved through an effective method called the $\exp (-\phi(\epsilon))$-expansion method. As a consequence, a number of exact solutions to the fractional forms of EW and modified EW equations are generated.
\end{abstract}

Keywords: Fractional forms of EW and modified EW equations; Conformable sense; $\exp (-\phi(\epsilon))$-expansion method; Exact solutions

\section{Introduction}

These days, the fractional differential equations (FDEs) have been the subject of a lot of research, owing to their frequent appearance in various areas from physics and chemistry to biology and engineering. A variety of useful methods, such as sub-equation method [1-4], modified trial equation method [5-8], $\left(G^{\prime} / G\right)$-expansion method [912], exp-function method [13-16], Kudryashov method [1720], and first integral method [21-24] have been applied to find the exact solutions of FDEs. One capable technique which has newly gained special interest is the $\exp (-\phi(\epsilon))$ method. For instance, Hosseini et al. [25] exerted the $\exp (-\phi(\epsilon))$ method to produce the exact so-

\footnotetext{
*Corresponding Author: K. Hosseini, Department of Mechanical Engineering, Ahrar Institute of Technology and Higher Education, Rasht, Iran, E-mail: kamyar_hosseini@yahoo.com

A. Bekir, Department of Mathematics and Computer, Art-Science Faculty, Eskisehir Osmangazi University, Eskisehir, Turkey F. Rabiei, Department of Mathematics, Faculty of Science, Universiti Putra Malaysia, 43400 UPM Serdang, Selangor, Malaysia
}

lutions of the density-dependent conformable fractional diffusion-reaction equation and Raza et al. [26] adopted the $\exp (-\phi(\epsilon))$ method to extract the explicit solutions of higher dimensional equations with fractional temporal evolution. For more articles, see [27-40]. In this paper, the exact solutions of the fractional forms of $\mathrm{EW}$ and modified EW equations in the conformable sense are achieved by means of the $\exp (-\phi(\epsilon))$ method. The mathematical modelings of these fractional differential equations are presented below:

- $\quad$ The fractional EW equation [41]

$$
\begin{aligned}
& D_{t}^{\alpha} u(x, t)+\sigma D_{x}^{\alpha} u^{2}(x, t)-\delta D_{x x t}^{3 \alpha} u(x, t)=0, \\
& t>0, \quad 0<\alpha \leqslant 1 .
\end{aligned}
$$

- $\quad$ The fractional modified EW equation [41]

$$
\begin{aligned}
& D_{t}^{\alpha} u(x, t)+\sigma D_{x}^{\alpha} u^{3}(x, t)-\delta D_{x x t}^{3 \alpha} u(x, t)=0, \\
& t>0, \quad 0<\alpha \leqslant 1 .
\end{aligned}
$$

The EW equations describe the propagation of the wave in nonlinear media [42]. Several schemes have already been exerted for studying the above models; for example, Kudryashov method [17], ansatz method [41], and Fan sub-equation method [43]. This paper is organized as below: In Section 2, we will introduce the conformable derivative and its properties. In Section 3, we will explain the ideas of the $\exp (-\phi(\epsilon))$ method. In Section 4 , we will employ the $\exp (-\phi(\epsilon))$ method to solve the fractional forms of EW and modified EW equations; and at the end, we will provide the results.

\section{Conformable fractional derivative}

Recently, a new version of fractional derivatives "the conformable fractional derivative" was proposed in [44] which obeys some classical properties that cannot be satisfied by the other definitions [45]. The conformable fractional 
derivative of $f$ of order $\alpha$ is defined as [44]

$$
T_{\alpha}(f)(t)=\lim _{\tau \rightarrow 0} \frac{f\left(t+\tau t^{1-\alpha}\right)-f(t)}{\tau},
$$

where $f:(0, \infty) \rightarrow R, t>0$, and $\alpha \in(0,1]$. Some worthwhile features of the conformable derivative are as follows

$$
T_{\alpha}(a f+b g)=a T_{\alpha}(f)+b T_{\alpha}(g), \forall a, b \in R .
$$

$$
T_{\alpha}\left(t^{\mu}\right)=\mu t^{\mu-\alpha}, \forall \mu \in R .
$$

(iii)

$$
T_{\alpha}(f \circ g)(t)=t^{1-\alpha} g^{\prime}(t) f^{\prime}(g(t)) .
$$

\section{Basic ideas of $\exp (-\phi(\epsilon))$-expansion method}

Let's consider a nonlinear space-time FDE in the conformable sense as follows

$$
\begin{aligned}
& F\left(u, D_{t}^{\alpha_{1}} u, D_{x}^{\alpha_{2}} u, D_{t}^{2 \alpha_{1}} u, D_{t}^{\alpha_{1}} D_{x}^{\alpha_{2}} u, D_{x}^{2 \alpha_{2}} u, \ldots\right)=0, \\
& \quad 0<\alpha_{1}, \alpha_{2}<1 .
\end{aligned}
$$

By using the transformation

$$
u(x, t)=f(\epsilon), \quad \epsilon=k \frac{x^{\alpha_{2}}}{\alpha_{2}}-l \frac{t^{\alpha_{1}}}{\alpha_{1}},
$$

Eq. (3) changes into an ODE of integer order as

$$
G\left(f, f^{\prime}, f^{\prime \prime}, \ldots\right)=0,
$$

where $G$ is a function in the unknown function $f$ and its derivatives.

Let present the solution of Eq. (4) as below

$$
f(\epsilon)=\sum_{n=0}^{N} a_{n}(\exp (-\phi(\epsilon)))^{n},
$$

where $a_{n}, n=0,1, \ldots, N\left(a_{N} \neq 0\right)$ are unknown constants and $\phi(\epsilon)$ satisfies a nonlinear ordinary differential equation as

$$
\phi^{\prime}(\epsilon)=\exp (-\phi(\epsilon))+\mu \exp (\phi(\epsilon))+\lambda .
$$

Now, various cases can be considered:

Case 1. If $\lambda^{2}-4 \mu>0$ and $\mu \neq 0$, then

$$
\phi_{1}(\epsilon)=\ln \left(\frac{-\sqrt{\lambda^{2}-4 \mu} \tanh \left(\frac{\sqrt{\lambda^{2}-4 \mu}}{2}(\epsilon+C)\right)-\lambda}{2 \mu}\right) .
$$

Case 2. If $\lambda^{2}-4 \mu>0, \mu=0$, and $\lambda \neq 0$, then

$$
\phi_{2}(\epsilon)=-\ln \left(\frac{\lambda}{\cosh (\lambda(\epsilon+C))+\sinh (\lambda(\epsilon+C))-1}\right) .
$$

Case 3. If $\lambda^{2}-4 \mu<0$ and $\mu \neq 0$, then

$$
\phi_{3}(\epsilon)=\ln \left(\frac{\sqrt{4 \mu-\lambda^{2}} \tan \left(\frac{\sqrt{4 \mu-\lambda^{2}}}{2}(\epsilon+C)\right)-\lambda}{2 \mu}\right) .
$$

To procure the positive integer $N$ in Eq. (5), we balance the terms in Eq. (4). Setting Eq. (5) in Eq. (4), results in

$$
P(\exp (-\phi(\epsilon)))=0 .
$$

Through equating all the coefficients in (6) to zero, we will attain an algebraic set, which can be easily solved for determining the unknowns. Substituting them into (5), finally yields the exact solutions of original Eq. (3).

\section{Applications}

Now, we adopt the $\exp (-\phi(\epsilon))$ method to seek the exact solutions of the fractional forms of EW and modified EW equations in the conformable sense.

\subsection{The conformable space-time fractional EW equation}

By using the transformation

$$
u(x, t)=f(\epsilon), \quad \epsilon=k \frac{x^{\alpha}}{\alpha}-l \frac{t^{\alpha}}{\alpha},
$$

Eq. (1) can be changed into the following ODE

$$
-l f^{\prime}+\sigma k\left(f^{2}\right)^{\prime}+\delta l k^{2} f^{\prime \prime \prime}=0 .
$$

Integrating (7) once with respect to $\epsilon$, gives

$$
-l f+\sigma k f^{2}+\delta l k^{2} f^{\prime \prime}=0,
$$

where integrating constant is supposed to be zero.

\subsubsection{Applying the $\exp (-\phi(\epsilon))$-expansion method}

By balancing $f^{2}$ and $f^{\prime \prime}$ in Eq. (8), we obtain $N=2$. Hence, Eq. (8) has the following formal solution

$$
f(\epsilon)=a_{0}+a_{1} \exp (-\phi(\epsilon))+a_{2} \exp (-2 \phi(\epsilon)) .
$$


Through setting Eq. (9) in Eq. (8) and equating all the coefficients to zero, we will achieve an algebraic set in the form

$$
\begin{aligned}
& -l a_{0}+\delta l \lambda \mu k^{2} a_{1}+2 \delta l \mu^{2} k^{2} a_{2}+k \sigma a_{0}^{2}=0, \\
& \left(\delta l \lambda^{2} k^{2}+2 \delta l \mu k^{2}-l\right) a_{1}+6 \delta l \lambda \mu k^{2} a_{2}+2 k \sigma a_{0} a_{1}=0, \\
& 3 \delta l \lambda k^{2} a_{1}+\left(4 \delta l \lambda^{2} k^{2}+8 \delta l \mu k^{2}-l\right) a_{2}+k \sigma a_{1}^{2}+2 k \sigma a_{0} a_{2}=0, \\
& 2 \delta l k^{2} a_{1}+10 \delta l \lambda k^{2} a_{2}+2 k \sigma a_{1} a_{2}=0, \\
& 6 \delta l k^{2} a_{2}+\delta \sigma a_{2}^{2}=0 .
\end{aligned}
$$

Applying the symbolic computation package, results in

Case 1.

$$
\begin{aligned}
& a_{0}=\mp \frac{6 l \mu \sqrt{\delta\left(\lambda^{2}-4 \mu\right)}}{\sigma\left(\lambda^{2}-4 \mu\right)}, a_{1}=\mp \frac{6 \delta l \lambda}{\sigma \sqrt{\delta\left(\lambda^{2}-4 \mu\right)}}, \\
& a_{2}=\mp \frac{6 \delta l}{\sigma \sqrt{\delta\left(\lambda^{2}-4 \mu\right)}}, k= \pm \frac{1}{\sqrt{\delta\left(\lambda^{2}-4 \mu\right)}} .
\end{aligned}
$$

Thus, the exact solutions to the fractional form of EW equation in the conformable sense can be constructed as follows

$$
\begin{aligned}
& \text { For } \lambda^{2}-4 \mu>0 \text { and } \mu \neq 0 \\
& \begin{aligned}
u_{1,2}(x, t)=\mp \frac{6 l \mu \sqrt{\delta\left(\lambda^{2}-4 \mu\right)}}{\sigma\left(\lambda^{2}-4 \mu\right)} \pm \frac{12 \delta l \lambda \mu}{\sigma \sqrt{\delta\left(\lambda^{2}-4 \mu\right)}\left(\sqrt{\lambda^{2}-4 \mu} \tanh \left(\frac{\sqrt{\lambda^{2}-4 \mu}}{2}\left( \pm \frac{1}{\sqrt{\delta\left(\lambda^{2}-4 \mu\right)}} \frac{\chi^{\alpha}}{\alpha}-l \frac{t^{\alpha}}{\alpha}+C\right)\right)+\lambda\right)} \\
\mp \frac{24 \delta l \mu^{2}}{\sigma \sqrt{\delta\left(\lambda^{2}-4 \mu\right)}\left(\sqrt{\lambda^{2}-4 \mu} \tanh \left(\frac{\sqrt{\lambda^{2}-4 \mu}}{2}\left( \pm \frac{1}{\left.\sqrt{\delta\left(\lambda^{2}-4 \mu\right.}\right)} \frac{\chi^{\alpha}}{\alpha}-l \frac{t^{\alpha}}{\alpha}+C\right)\right)+\lambda\right)^{2}} .
\end{aligned}
\end{aligned}
$$

For $\lambda^{2}-4 \mu>0, \mu=0$, and $\lambda \neq 0$

$$
\begin{gathered}
u_{3,4}(x, t)=\mp \frac{6 \delta l \lambda^{2}}{\sigma \sqrt{\delta \lambda^{2}}\left(\cosh \left(\lambda\left( \pm \frac{1}{\sqrt{\delta \lambda^{2}}} \frac{\chi^{\alpha}}{\alpha}-l \frac{t^{\alpha}}{\alpha}+C\right)\right)+\sinh \left(\lambda\left( \pm \frac{1}{\sqrt{\delta \lambda^{2}}} \frac{\chi^{\alpha}}{\alpha}-l \frac{t^{\alpha}}{\alpha}+C\right)\right)-1\right)} \\
\mp \frac{6 \delta l \lambda^{2}}{\sigma \sqrt{\delta \lambda^{2}}\left(\cosh \left(\lambda\left( \pm \frac{1}{\sqrt{\delta \lambda^{2}}} \frac{\chi^{\alpha}}{\alpha}-l \frac{t^{\alpha}}{\alpha}+C\right)\right)+\sinh \left(\lambda\left( \pm \frac{1}{\sqrt{\delta \lambda^{2}}} \frac{\chi^{\alpha}}{\alpha}-l \frac{t^{\alpha}}{\alpha}+C\right)\right)-1\right)^{2}} .
\end{gathered}
$$

For $\lambda^{2}-4 \mu<0$ and $\mu \neq 0$

$$
\begin{gathered}
u_{5,6}(x, t)=\mp \frac{6 l \mu \sqrt{\delta\left(\lambda^{2}-4 \mu\right)}}{\sigma\left(\lambda^{2}-4 \mu\right)} \pm \frac{12 \delta l \lambda \mu}{\sigma \sqrt{\delta\left(\lambda^{2}-4 \mu\right)}\left(\sqrt{4 \mu-\lambda^{2}} \tan \left(\frac{\sqrt{4 \mu-\lambda^{2}}}{2}\left( \pm \frac{1}{\sqrt{\delta\left(\lambda^{2}-4 \mu\right)}} \frac{x^{\alpha}}{\alpha}-l \frac{t^{\alpha}}{\alpha}+C\right)\right)+\lambda\right)} \\
\mp \frac{24 \delta l \mu^{2}}{\sigma \sqrt{\delta\left(\lambda^{2}-4 \mu\right)}\left(\sqrt{4 \mu-\lambda^{2}} \tan \left(\frac{\sqrt{4 \mu-\lambda^{2}}}{2}\left( \pm \frac{1}{\sqrt{\delta\left(\lambda^{2}-4 \mu\right)}} \frac{\chi^{\alpha}}{\alpha}-l \frac{t^{\alpha}}{\alpha}+C\right)\right)+\lambda\right)^{2}} .
\end{gathered}
$$

Case 2.

$$
a_{0}= \pm \frac{l\left(\lambda^{2}+2 \mu\right) \sqrt{-\delta\left(\lambda^{2}-4 \mu\right)}}{\sigma\left(\lambda^{2}-4 \mu\right)}, \quad a_{1}=\mp \frac{6 \delta l \lambda}{\sigma \sqrt{-\delta\left(\lambda^{2}-4 \mu\right)}}, \quad a_{2}=\mp \frac{6 \delta l}{\sigma \sqrt{-\delta\left(\lambda^{2}-4 \mu\right)}}, \quad k= \pm \frac{1}{\sqrt{-\delta\left(\lambda^{2}-4 \mu\right)}} .
$$

Consequently, the exact solutions to the fractional form of EW equation in the conformable sense can be established as follows 
For $\lambda^{2}-4 \mu>0$ and $\mu \neq 0$

$$
\begin{gathered}
u_{7,8}(x, t)= \pm \frac{l\left(\lambda^{2}+2 \mu\right) \sqrt{-\delta\left(\lambda^{2}-4 \mu\right)}}{\sigma\left(\lambda^{2}-4 \mu\right)} \pm \frac{12 \delta l \lambda \mu}{\sigma \sqrt{-\delta\left(\lambda^{2}-4 \mu\right)}\left(\sqrt { \lambda ^ { 2 } - 4 \mu } \operatorname { t a n h } \left(\frac { \sqrt { \lambda ^ { 2 } - 4 \mu } } { 2 } \left( \pm \frac{1}{\left.\left.\left.\sqrt{-\delta\left(\lambda^{2}-4 \mu\right)} \frac{x^{\alpha}}{\alpha}-l \frac{t^{\alpha}}{\alpha}+C\right)\right)+\lambda\right)}\right.\right.\right.} \\
\mp \frac{24 \delta l \mu^{2}}{\sigma \sqrt{-\delta\left(\lambda^{2}-4 \mu\right)}\left(\sqrt{\lambda^{2}-4 \mu} \tanh \left(\frac{\sqrt{\lambda^{2}-4 \mu}}{2}\left( \pm \frac{1}{\sqrt{-\delta\left(\lambda^{2}-4 \mu\right)}} \frac{x^{\alpha}}{\alpha}-l \frac{t^{\alpha}}{\alpha}+C\right)\right)+\lambda\right)^{2}} \cdot
\end{gathered}
$$

For $\lambda^{2}-4 \mu>0, \mu=0$, and $\lambda \neq 0$

$$
\begin{aligned}
u_{9,10}(x, t) & = \pm \frac{l \sqrt{-\delta \lambda^{2}}}{\sigma} \mp \frac{6 \delta l \lambda^{2}}{\sigma \sqrt{-\delta \lambda^{2}}\left(\cosh \left(\lambda\left( \pm \frac{1}{\sqrt{-\delta \lambda^{2}}} \frac{\chi^{\alpha}}{\alpha}-l \frac{t^{\alpha}}{\alpha}+C\right)\right)+\sinh \left(\lambda\left( \pm \frac{1}{\sqrt{-\delta \lambda^{2}}} \frac{\chi^{\alpha}}{\alpha}-l \frac{t^{\alpha}}{\alpha}+C\right)\right)-1\right)} \\
& \mp \frac{6 \delta l \lambda^{2}}{\sigma \sqrt{-\delta \lambda^{2}}\left(\cosh \left(\lambda\left( \pm \frac{1}{\sqrt{-\delta \lambda^{2}}} \frac{\chi^{\alpha}}{\alpha}-l \frac{t^{\alpha}}{\alpha}+C\right)\right)+\sinh \left(\lambda\left( \pm \frac{1}{\sqrt{-\delta \lambda^{2}}} \frac{\chi^{\alpha}}{\alpha}-l \frac{t^{\alpha}}{\alpha}+C\right)\right)-1\right)^{2}} .
\end{aligned}
$$

For $\lambda^{2}-4 \mu<0$ and $\mu \neq 0$

$$
\begin{gathered}
u_{11,12}(x, t)= \pm \frac{l\left(\lambda^{2}+2 \mu\right) \sqrt{-\delta\left(\lambda^{2}-4 \mu\right)}}{\sigma\left(\lambda^{2}-4 \mu\right)} \pm \frac{12 \delta l \lambda \mu}{\sigma \sqrt{-\delta\left(\lambda^{2}-4 \mu\right)}\left(\sqrt{4 \mu-\lambda^{2}} \tan \left(\frac{\sqrt{4 \mu-\lambda^{2}}}{2}\left( \pm \frac{1}{\sqrt{-\delta\left(\lambda^{2}-4 \mu\right)}} \frac{\chi^{\alpha}}{\alpha}-l \frac{t^{\alpha}}{\alpha}+C\right)\right)+\lambda\right)} \\
\mp \frac{24 \delta l \mu^{2}}{\sigma \sqrt{-\delta\left(\lambda^{2}-4 \mu\right)}\left(\sqrt{4 \mu-\lambda^{2}} \tan \left(\frac{\sqrt{4 \mu-\lambda^{2}}}{2}\left( \pm \frac{1}{\sqrt{-\delta\left(\lambda^{2}-4 \mu\right)}} \frac{x^{\alpha}}{\alpha}-l \frac{t^{\alpha}}{\alpha}+C\right)\right)+\lambda\right)^{2}} \cdot
\end{gathered}
$$

\subsection{The conformable space-time fractional modified EW equation}

By using the transformation

$$
u(x, t)=f(\epsilon), \quad \epsilon=k \frac{x^{\alpha}}{\alpha}-l \frac{t^{\alpha}}{\alpha},
$$

Eq. (2) can be converted to an ODE as follows

$$
-l f^{\prime}+\sigma k\left(f^{3}\right)^{\prime}+\delta l k^{2} f^{\prime \prime \prime}=0 .
$$

Integrating (10) once with respect to $\epsilon$, yields

$$
-l f+\sigma k f^{3}+\delta l k^{2} f^{\prime \prime}=0
$$

where integrating constant is assumed to be zero.

\subsubsection{Applying the $\exp (-\phi(\epsilon))$-expansion method}

By balancing $f^{3}$ and $f^{\prime \prime}$ in Eq. (11), we take $N=1$. Consequently, Eq. (11) has the following formal solution

$$
f(\epsilon)=a_{0}+a_{1} \exp (-\phi(\epsilon)) \text {. }
$$

By inserting Eq. (12) along with its necessary derivative in Eq. (11) and equating all the coefficients to zero, we will derive an algebraic set as

$$
\begin{aligned}
& -l a_{0}+\delta l \lambda \mu k^{2} a_{1}+k \sigma a_{0}^{3}=0, \\
& \left(\delta l \lambda^{2} k^{2}+2 \delta l \mu k^{2}-l\right) a_{1}+3 k \sigma a_{0}^{2} a_{1}=0, \\
& 3 \delta l \lambda k^{2} a_{1}+3 k \sigma a_{0} a_{1}^{2}=0, \\
& 2 \delta l k^{2} a_{1}+k \sigma a_{1}^{3}=0 .
\end{aligned}
$$


Using the symbolic computation package, we will find

$$
a_{0}=\frac{1}{2} \lambda a_{1}, \quad k= \pm \frac{2}{\sqrt{-2 \delta\left(\lambda^{2}-4 \mu\right)}}, \quad l=\mp \frac{\sigma a_{1}^{2} \sqrt{-2 \delta\left(\lambda^{2}-4 \mu\right)}}{4 \delta} .
$$

Thus, the exact solutions to the fractional form of modified EW equation in the conformable sense can be constructed as follows

For $\lambda^{2}-4 \mu>0$ and $\mu \neq 0$

$$
u_{1,2}(x, t)=\frac{1}{2} \lambda a_{1}-\frac{2 \mu a_{1}}{\sqrt{\lambda^{2}-4 \mu} \tanh \left(\frac{\sqrt{\lambda^{2}-4 \mu}}{2}\left( \pm \frac{2}{\sqrt{-2 \delta\left(\lambda^{2}-4 \mu\right)}} \frac{\chi^{\alpha}}{\alpha} \pm \frac{\sigma a_{1}^{2} \sqrt{-2 \delta\left(\lambda^{2}-4 \mu\right)}}{4 \delta} \frac{t^{\alpha}}{\alpha}+C\right)\right)+\lambda} .
$$

For $\lambda^{2}-4 \mu>0, \mu=0$, and $\lambda \neq 0$

$$
u_{3,4}(x, t)=\frac{1}{2} \lambda a_{1}+\frac{\lambda a_{1}}{\cosh \left(\lambda\left( \pm \frac{2}{\sqrt{-2 \delta \lambda^{2}}} \frac{\chi^{\alpha}}{\alpha} \pm \frac{\sigma a_{1}^{2} \sqrt{-2 \delta \lambda^{2}}}{4 \delta} \frac{t^{\alpha}}{\alpha}+C\right)\right)+\sinh \left(\lambda\left( \pm \frac{2}{\sqrt{-2 \delta \lambda^{2}}} \frac{\chi^{\alpha}}{\alpha} \pm \frac{\sigma a_{1}^{2} \sqrt{-2 \delta \lambda^{2}}}{4 \delta} \frac{t^{\alpha}}{\alpha}+C\right)\right)-1} .
$$

For $\lambda^{2}-4 \mu<0$ and $\mu \neq 0$

$$
u_{5,6}(x, t)=\frac{1}{2} \lambda a_{1}-\frac{2 \mu a_{1}}{\sqrt{4 \mu-\lambda^{2}} \tan \left(\frac{\sqrt{4 \mu-\lambda^{2}}}{2}\left( \pm \frac{2}{\sqrt{-2 \delta\left(\lambda^{2}-4 \mu\right)}} \frac{\chi^{\alpha}}{\alpha} \pm \frac{\sigma a_{1}^{2} \sqrt{-2 \delta\left(\lambda^{2}-4 \mu\right)}}{4 \delta} \frac{t^{\alpha}}{\alpha}+C\right)\right)+\lambda} .
$$

\section{Conclusion}

In this investigation, the fractional forms of EW and modified EW equations in the conformable sense were studied, successfully. First, by adopting a traveling wave transformation, the governing space-time fractional models were converted to the nonlinear ordinary differential equations; and then, the resulting NLODEs were solved using an effective method called the $\exp (-\phi(\epsilon))$-expansion method. As a consequence, a variety of exact solutions to the fractional forms of EW and modified EW equations were formally extracted; confirming the competence of the scheme.

\section{References}

[1] K.U. Tarigh, M. Younis, Bright, dark and other optical solitons with second order spatiotemporal dispersion, Optik 142 (2017) 446450.

[2] S.S. Afzal, M. Younis, S.T.R. Rizvi, Optical dark and dark-singular solitons with anti-cubic nonlinearity, Optik 147 (2017) $27-31$.

[3] N. Cheemaa, M. Younis, New and more general traveling wave solutions for nonlinear Schrödinger equation, Waves in Random and Complex Media 26 (2016) 30-41.

[4] M. Younis, H. ur Rehman, S.T.R. Rizvi, S.A. Mahmood, Dark and singular optical solitons perturbation with fractional temporal evolution, Superlattice and Microstructures 104 (2017) 525-531.

[5] H. Bulut, H.M. Baskonus, Y. Pandir, The modified trial equation method for fractional wave equation and time fractional generalized Burgers equation, Abstract and Applied Analysis 2013 (2013) 636802.

[6] Y.A. Tandogan, N. Bildik, Exact solutions of the time-fractional Fisher equation by using modified trial equation method, AIP Conference Proceedings 1738 (2016) 290018.

[7] M. Odabasi, E. Misirli, On the solutions of the nonlinear fractional differential equations via the modified trial equation method, Mathematical Methods in the Applied Sciences (2015), doi:10.1002/mma.3533.

[8] H. Bulut, Y. Pandir, Modified trial equation method to the nonlinear fractional Sharma-Tasso-Olever equation, International Journal of Modeling and Optimization 3 (2013) 353-357.

[9] D. Baleanu, Y. Ugurlu, M. Inc, B. Kilic, Improved exp(- $\phi(\epsilon))$-expansion method for the time-fractional biological population model and Cahn-Hilliard equation, Journal of Computational and Nonlinear Dynamics 10 (2015) 051016.

[10] S. Sahoo, S. Saha Ray, Solitary wave solutions for time fractional third order modified KdV equation using two reliable techniques $\exp (-\phi(\epsilon))$-expansion method and improved exp(- $\phi(\epsilon))$-expansion method, Physica A 448 (2016) 265-282. 
[11] A. Sardar, S.M. Husnine, S.T.R. Rizvi, M. Younis, K. Ali, Multiple travelling wave solutions for electrical transmission line model, Nonlinear Dynamics 82 (2015) 1317-1324.

[12] M. Younis, Optical solitons in (n+1)-dimensions with Kerr and power law nonlinearities, Modern Physics Letters B 31 (2017) 1750186.

[13] O. Guner, A. Bekir, H. Bilgil, A note on exp-function method combined with complex transform method applied to fractional differential equations, Advances in Nonlinear Analysis 4 (2015) 201-208.

[14] M. Ekici, M. Mirzazadeh, A. Sonmezoglu, Q. Zhou, H. Triki, M.Z. Ullah, S.P. Moshokoa, A. Biswas, Optical solitons in birefringent fibers with Kerr nonlinearity by exp-function method, Optik 131 (2017) 964-976.

[15] O. Guner, E. Aksoy, A. Bekir, A.C. Cevikel, Different methods for (3+1)-dimensional space-time fractional modified KdV-ZakharovKuznetsov equation, Computers and Mathematics with Applications 71 (2016) 1259-1269.

[16] A. Bekir, Ö. Güner, A.H. Behrawy, A. Biswas, Solving nonlinear differential equations using exp-function and $\exp (-\phi(\epsilon))$-expansion methods, Romanian Journal of Physics 60 (2015) 360-378.

[17] K. Hosseini, Z. Ayati, Exact solutions of space-time fractional EW and modified EW equations using Kudryashov method, Nonlinear Science Letters A 7 (2016) 58-66.

[18] A.H. Arnous, S.A. Mahmood, M. Younis, Dynamics of optical solitons in dual-core fibers via two integration schemes, Superlattice and Microstructures 106 (2017) 156-162.

[19] K. Hosseini, R. Ansari, New exact solutions of nonlinear conformable time-fractional Boussinesq equations using the modified Kudryashov method, Waves in Random and Complex Media 27 (2017) 628-636.

[20] K. Hosseini, E. Yazdani Bejarbaneh, A. Bekir, M. Kaplan, New exact solutions of some nonlinear evolution equations of pseudoparabolic type, Optical and Quantum Electronics 49 (2017) 241.

[21] M. Mirzazadeh, M. Eslami, A. Biswas, Solitons and periodic solutions to a couple of fractional nonlinear evolution equations, Pramana Journal of Physics 82 (2014) 465-476.

[22] M. Eslami, B. Fathi Vajargah, M. Mirzazadeh, A. Biswas, Application of first integral method to fractional partial differential equations, Indian Journal of Physiscs 88 (2014) 177-184.

[23] Y. Çenesiz, D. Baleanu, A. Kurt, O. Tasbozan, New exact solutions of Burgers' type equations with conformable derivative, Waves in Random and Complex Media (2016), doi:10.1080/17455030.2016.1205237.

[24] M. Ekici, M. Mirzazadeh, M. Eslami, Q. Zhou, S.P. Moshokoa, A. Biswas, M. Belic, Optical soliton perturbation with fractional-temporal evolution by first integral method with conformable fractional derivatives, Optik 127 (2016) 10659-10669.

[25] K. Hosseini, P. Mayeli, A. Bekir, O. Guner, Density-dependent conformable space-time fractional diffusion-reaction equation and its exact solutions, Communication in Theoretical Physics 69 (2018) 1-4.

[26] N. Raza, S. Sial, M. Kaplan, Exact periodic and explicit solutions of higher dimensional equations with fractional temporal evolution, Optik 156 (2018) 628-634.

[27] M. Mirzazadeh, E. Ekici, Q. Zhou, A. Sonmezoglu, Analytical study of solitons in the fiber waveguide with power law nonlinearity, Superlattices and Microstructures 101 (2017) 493-506.

[28] S.M.R. Islam, K. Khan, M.A. Akbar, Exact solutions of unsteady Korteweg-de Vries and time regularized long wave equations, Springer Plus 4 (2015) 124.

[29] M.G. Hafez, M.A. Akbar, An exponential expansion method and its application to the strain wave equation in microstructured solids, Ain Shams Engineering Journal 6 (2015) 683-690.

[30] K. Hosseini, A. Bekir, M. Kaplan, Ö. Güner, On a new technique for solving the nonlinear conformable time-fractional differential equations, Optical and Quantum Electronics 49 (2017) 343.

[31] K. Hosseini, Y.J. Xu, P. Mayeli, A. Bekir, P. Yao, Q. Zhou, Ö. Güner, A study on the conformable time-fractional Klein-Gordon equations with quadratic and cubic nonlinearities, Optoelectronics and Advanced Materials-Rapid Communications 11 (2017) 423-429.

[32] K. Hosseini, A. Bekir, R. Ansari, Exact solutions of nonlinear conformable time-fractional Boussinesq equations using the exp(- $\phi(\epsilon))$ expansion method, Optical and Quantum Electronics 49 (2017) 131.

[33] A. Korkmaz, K. Hosseini, Exact solutions of a nonlinear conformable time-fractional parabolic equation with exponential nonlinearity using reliable methods, Optical and Quantum Electronics 49 (2017) 278.

[34] N. Taghizadeh, Q. Zhou, M. Ekici, M. Mirzazadeh, Soliton solutions for Davydov solitons in $\alpha$-helix proteins, Superlattices and Microstructures 102 (2017) 323-341.

[35] M. Ekici, Q. Zhou, A. Sonmezoglu, J. Manafian, M. Mirzazadeh, The analytical study of solitons to the nonlinear Schrödinger equation with resonant nonlinearity, Optik (2017) 378-382.

[36] S. Ali, S.T.R. Rizvi, M. Younis, Traveling wave solutions for nonlinear dispersive water-wave systems with time-dependent coefficients, Nonlinear Dynamics 82 (2015) 1755-1762.

[37] R. Ashraf, M.O. Ahmad, M. Younis, K. Ali, S.T.R. Rizvi, Dipole and Gausson soliton for ultrashort laser pulse with high order dispersion, Superlattice and Microstructures 109 (2017) 504-510.

[38] W. Islam, H. ur Rehman, M. Younis, Weakly nonlocal single and combined solitons in nonlinear optics with cubic quintic nonlinearities, Journal of Nanoelectronics and Optoelectronics 12 (2017) 1008-1012.

[39] M. Younis, S.T.R. Rizvi, Optical soliton like-pulses in ring-cavity fibers of carbon nanotubes, Journal of Nanoelectronics and Optoelectronics 11 (2016) 276-279.

[40] N. Cheemaa, M. Younis, New and more exact traveling wave solutions to integrable (2+1)-dimensional Maccari system, Nonlinear Dynamics 83 (2016) 1395-1401.

[41] A. Korkmaz, Exact solutions of space-time fractional EW and modified EW equations, arXiv:1601.01294v1 [nlin.SI] 6 Jan 2016. 
[42] S.B.G. Karakoc T. Geyikli, Numerical solution of the modified equal width wave equation, International Journal of Differential Equations 2012 (2012) 587208.

[43] H. Jafari, H. Tajadodi, D. Baleanu, Application of a homogeneous balance method to exact solutions of nonlinear fractional evolution equations, Journal of Computational and Nonlinear Dynamics 9 (2014) 021019.

[44] R. Khalil, M. Al-Horani, A. Yousef, M. Sababheh, A new definition of fractional derivative, Journal of Computational and Applied Mathematics 264 (2014) 65-70.

[45] M. Eslami, H. Rezazadeh, The first integral method for Wu-Zhang system with conformable time-fractional derivative, Calcolo 53 (2016) 475-485. 